Table 1.

\begin{tabular}{|c|c|c|c|c|c|}
\hline & $\begin{array}{l}\text { JIA-U } \\
n=49\end{array}$ & $\begin{array}{l}\text { Idiopathic uveitis } \\
\qquad \mathrm{n}=14\end{array}$ & $\begin{array}{c}\text { JIA } \\
\mathrm{n}=37\end{array}$ & $p$-value ${ }^{*}$ & $p$-value ${ }^{\#}$ \\
\hline Total score & $5.0(3.0-11.0)$ & $4.5(2-9.8)$ & $2.0(0-4.0)$ & 0.5739 & $<0.0001$ \\
\hline VF score & $2.0(0-3.0)$ & $1.0(0-3.0)$ & $0(0-0)$ & 0.9098 & $<0.0001$ \\
\hline QoL score & $3.0(2.0-6.0)$ & $3.5(2.0-4.8)$ & $2.0(0-4.0)$ & 0.5611 & 0.0005 \\
\hline
\end{tabular}

Numbers are medians (IQR). * JIA-U vs idiopathic uveitis; "JIA-U vs JIA

Disclosure of Interests: None declared

DOI: 10.1136/annrheumdis-2021-eular.2994

\section{POS1317 PREDICTIVE FACTORS FOR RESPONSE TO TREATMENT IN A LONG-TERM COHORT OF PATIENTS WITH JUVENILE IDIOPATHIC ARTHRITIS-ASSOCIATED UVEITIS}

L. Marelli ${ }^{1}$, F. Minoia ${ }^{2}$, G. Beretta ${ }^{2}$, C. Mapelli ${ }^{3}$, G. Leone ${ }^{3}$, G. Cincinelli ${ }^{4}$, T. Giani ${ }^{5}$, P. Nucci ${ }^{6}$, R. Cimaz ${ }^{4,6}$, E. Miserocchi ${ }^{7} .{ }^{1}$ Ospedale San Giuseppe, IRCCS Multimedica, Ophthalmology, Milan, Italy; ${ }^{2}$ Fondazione IRCCS Ca' Granda Ospedale Maggiore Policlinico, Pediatric Rheumatology, Milan, Italy; ${ }^{3}$ Fondazione IRCCS Ca' Granda Ospedale Maggiore Policlinico, Ophthalmology, Milan, Italy; ${ }^{4}$ ASST Gaetano Pini CTO, Pediatric Rheumatology, Milan, Italy; ${ }^{5}$ Università degli Studi di Siena, Pediatric Rheumatology, Siena, Italy; ${ }^{6}$ Università degli Studi di Milano, Dipartimento di Scienze Cliniche e di Comunità, Milan, Italy; ${ }^{7}$ IRCCS Ospedale San Raffaele, Ophthalmology, Milan, Italy

Background: Uveitis is the main extraarticular complication of juvenile idiopathic arthritis (JIA) with still a significant impact on JIA morbidity, despite continuous improvement in systemic treatment. Although antinuclear antibody positivity and early onset of JIA have been associated with a high risk of uveitis onset, so far no clinical features have been widely recognized as predictive factors for JIA-associated uveitis (JIA-U) response to treatment.

Objectives: To investigate clinical features associated with response to systemic treatment in a long-term cohort of patients with JIA-U

Methods: Clinical records of patients with JIA-U were retrospectively reviewed with regard to clinical features, therapeutic choices and outcome. Clinical and laboratory variables were compared by means of Mann-Whitney $\mathrm{U}$ test or chisquare/Fisher exact test, as appropriate.

Results: Data from $164 \mathrm{JIA}-\mathrm{U}$ patients were analysed (81.7\% female), with a median follow up of 12.1 years (7.1-17.3). Median age at JIA and uveitis onset was $2.6(1.6-4.8)$ and $4.8(2.9-7.0)$ years, respectively. Monotherapy with a conventional disease-modifying antirheumatic drug (DMARD) was used in $25.0 \%$ of patients, while 111 patients $(67.7 \%)$ received at least one biologic DMARD (bDMARDs). Compared to patients responsive to DMARDs, children requiring a bDMARDs for uveitis had a lower median age at both JIA (2.4 vs 4.3 years, $p$ 0.0234 ) and uveitis onset (4.1 vs 6.2 years, p 0.0023). Despite no differences in ocular damage at onset and median disease duration, patients not responsive to conventional DMARDs showed a higher frequency of ocular damage at the last visit ( $66.2 \%$ vs $33.3 \%, p$ 0.011). Children requiring more than one bDMARD for uveitis presented a more frequent polyarticular course $(87.0 \%$ vs $20.2 \%$, p 0.0022), a longer disease duration (median follow-up: 14.2 vs 10.4 years, $p$ 0.0397 ) and a higher frequency of visual loss (best corrected visual acuity $<4 / 10$ : $23.3 \%$ vs $6.3 \%$, p 0.0069).

Conclusion: JIA-U patients with a lack of response to conventional DMARDs were significantly younger both at JIA and uveitis onset. Severe JIA-U requiring more than one bDMARDs was associated with polyarticular JIA course and longer disease duration. Children resistant to conventional treatment need prompt recognition and additional strategies to improve long-term outcome.

REFERENCES:

[1] Heiligenhaus et al. Predictive factors and biomarkers for the 2-year outcome of uveitis in juvenile idiopathic arthritis. Rheumatology 2019.

Disclosure of Interests: None declared

DOI: 10.1136/annrheumdis-2021-eular.3006

\section{POS1318 A NATIONWIDE COHORT STUDY ON CLINICAL AND LABORATORY MANIFESTATIONS IN CHILDREN WITH MULTISYSTEM INFLAMMATORY SYNDROME (MIS-C)}

M. Zajc Avramovic ${ }^{1,2}$, K. Vincek ${ }^{3}$, G. Mlakar ${ }^{4}$, N. Emersic ${ }^{1}$, T. Plankar Srovin $^{3}$ T. Avsic-Zupanc ${ }^{5}$, A. Ihan ${ }^{5}$, T. Avcin ${ }^{1,2} .{ }^{1}$ University Medical Center Ljubljana, Children's Hospital, Department for Allergology, Rheumatology and Clinical
Immunology, Ljubljana, Slovenia; ${ }^{2}$ University of Ljubljana, Faculty of Medicine, Pediatrics, Ljubljana, Slovenia; ${ }^{3}$ University Medical Center Ljubljana, Clinic of Infectious Diseases, Ljubljana, Slovenia; ${ }^{4}$ University Medical Center Ljubljana, Children's Hospital, Department for Cardiology, Ljubljana, Slovenia; ${ }^{5}$ University of Ljubljana, Faculty of Medicine, Institute of Microbiology and Immunology, Ljubljana, Slovenia

Background: Multisystem inflammatory syndrome in children (MIS-C) was recognized during the 2020 pandemic of SARS-CoV-2. Because of the relative rarity current knowledge is limited, especially in the European Caucasian population.

Objectives: To report the epidemiology, clinical and laboratory characteristics of patients with MIS-C in a nationwide cohort study in Slovenia.

Methods: This is a nationwide prospective cohort study of all consecutive patients with MIS-C, admitted from the beginning of epidemics to $31 \mathrm{st}$ December 2020 to University Medical Centre Ljubljana, Slovenia, the only tertiary care pediatric rheumatology center in the country. The inclusion criteria were meeting the CDC criteria for MIS-C. Infection with SARS-CoV-2 was confirmed in all patients by positive antibodies for SARS-CoV-2. Data were collected from the patients' medical records. Data on the COVID-19 epidemics in Slovenia were collected from National Institute of Public Health. Population data were provided by the Statistical Office of the Republic of Slovenia.

Results: Twenty-three patients with MIS-C were diagnosed nationwide in Slovenia, all of them in the second wave of epidemics from 14th September to 31st December 2020. All patients were Caucasian and the estimated prevalence of MIS-C was 5.8/100 000 persons younger than 19 years of age. Detailed analyses were available in 20 patients of which 14 were boys $(70$ $\%$ ), median age was 12.4 years (4 months to 17.7 years). Two patients (10\%) were treated in the intensive care unit and none of the patients died. Troponin was elevated in 15/20 (75\%) patients during the disease course, and $7 / 15$ $(47 \%)$ of these had normal troponin level at admission. The serum level of troponin closely followed the serum level of CRP. Six out of 20 (30\%) patients had elevated pancreatic enzymes in the second week of the disease after treatment was already given, and one patient developed asymptomatic acute pancreatitis with serum lipase level reaching the maximum of $25 \mu \mathrm{kat} / \mathrm{L}$. Al patients had elevated levels of D-dimer with no signs of thrombosis. Five patients $(5 / 20 ; 25 \%)$ had pleural effusions and five patients $(5 / 20,25 \%)$ had ascites. Half of the patients $(10 / 20 ; 50 \%)$ had hepatosplenomegaly and eight $(8 / 20 ; 40 \%)$ had mesenterial lymphadenopathy. Three patients $(3 / 20$ $15 \%)$ had radiologic signs of cholecystitis. Two patients had thickened lung parenchyma. All patients received IVIG and systemic glucocorticosteroids. Because of resistant or organ threatening disease 4 patients $(4 / 20,20 \%)$ received high dose methylprednisolone pulse therapy. Biologic therapy with anakinra was started in 2 patients. Nineteen patients (19/20,95\%) received acetylsalicylic acid and prophylactic anticoagulation was prescribed in 15/20 (75\%) of patients.

The mean follow up was 50 days $(14-122)$. At the last follow-up visit all patients had normal laboratory parameters of inflammation, troponin, pro-BNP, d-dimer values and normal heart function.

Table 1. Clinical characteristics.

\begin{tabular}{ll}
\hline & \\
\hline Fever n (\%) & \\
Fever duration (days) [ min; max] & $20(100)$ \\
Headache & $5.9[4 ; 8]$ \\
Lymphadenopathy & $8(40)$ \\
Chest pain & $15(75)$ \\
Tachycardia & $6(30)$ \\
Gastrointestinal involvement & $16(80)$ \\
- Abdominal pain & $19(95)$ \\
- Vomiting & $16(80)$ \\
- Diarrhoea & $11(55)$ \\
- Loss of apetite & $9(45)$ \\
Cough & $18(90)$ \\
Skin and mucous involvement & $7(35)$ \\
- Rash & $14(70)$ \\
• Palmar/plantar oedema & $12(60)$ \\
- Lip and mouth changes & $3(15)$ \\
• Bilateral conjunctivitis & $12(60)$ \\
Myocarditis & $14(70)$ \\
\hline
\end{tabular}

Conclusion. A very high incidence of MIS-C, estimated 5.8/100 000 persons under the age of 19 with a predominantly cardiac involvement but very good outcome was noted in European Caucasian population in a nationwide cohort study in Slovenia. Attention to newly described pancreatic involvement should be raised. 

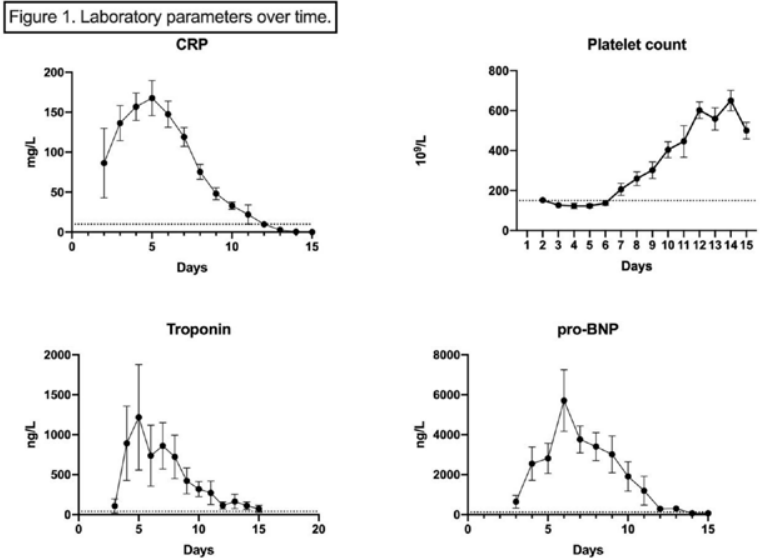

LDH
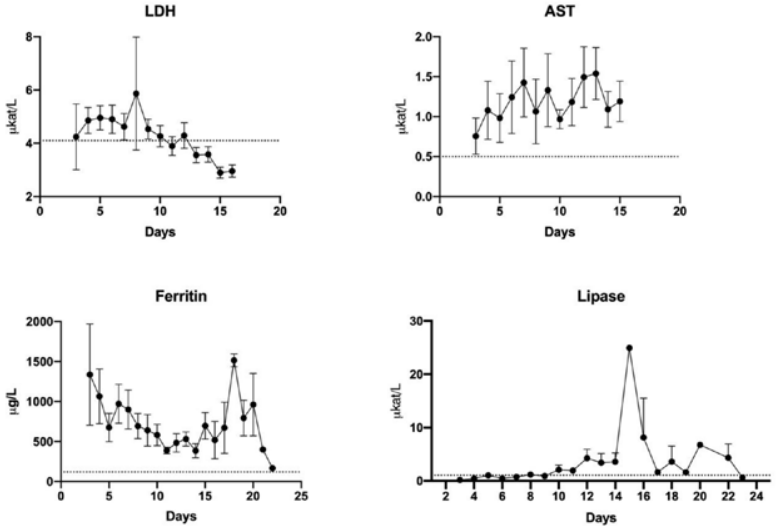

Normal values are shown in dotted line. Lipase is shown only in patients with at least one pathological value.

Conclusion: A very high incidence of MIS-C, estimated 5.8/100 000 persons under the age of 19 with a predominantly cardiac involvement but very good outcome was noted in European Caucasian population in a nationwide cohort study in Slovenia. Attention to newly described pancreatic involvement should be raised.

Disclosure of Interests: None declared

DOI: 10.1136/annrheumdis-2021-eular.3022

\section{POS1319 RAYNAUD'S PHENOMENON IN A SINGLE CENTER COHORT OF TURKISH CHILDREN}

S. Çağlayan ${ }^{1}$, F. Demir ${ }^{1}$, B. Sancar ${ }^{1}$, K. Ulu' ${ }^{1}$, T. Coşkuner ${ }^{1}$, B. Sözeri' ${ }^{1}{ }^{1}$ Health Sciences University, Umraniye Traning and Research Hospita, Pediatric Rheumatology, Istanbul, Turkey

Background: Raynaud's phenomenon (RF) is a vasospastic condition characterized by episodic color changes of blanching, cyanosis, and hyperemia of the extremities of the body

Objectives: We aim to examine the clinical presentation, capillaroscopic findings and disease associations of Raynaud's phenomenon (RP) in pediatric patients Methods: We retrospectively enrolled a single-center cohort of 247 consecutive pediatric patients with RP admitted to Umraniye Training and Research Hospital, Pediatric Rheumatology Clinic, Istanbul, Turkey, since 2016. Medical records were analyzed for clinical presentation, disease associations, and physical examination and laboratory findings.

Results: We reported 247 patients (152 female, 95 male) with RP. Their mean age at disease onset was $14.5 \pm 2.55$ years and median age at diagnosis was $15.4 \pm 2.21$ years. In patients with secondary RP syndrome $(10.9 \%)$, the age of complaint was found to be lower than others $(p=0.03)$. Although most patients have affected upper extremity (hand and fingers), lower extremity (toes) involvement was also detected in $50(20 \%)$ patients. In both the patients with primary and secondary RP, main triggering factor was found as cold exposure. Biphasic (53.6\%) color changes were more seen in primary $\mathrm{RP}$, although triphasic $(51.8 \%)$ color changes were more in patients with secondary RP $(p=0.00)$. The presence of antinuclear antibodies and abnormal nailfold capillaries were more seen in patients with secondary RP (28\% vs $74 \%$ and $35.3 \%$ vs 66.7 , respectively $p=0.00$ ). Digital ulcer was detected $5.2 \%$ of patients (mostly secondary). Out of 247,31 patients were treated with lowdose aspirin, 47 with calcium blockers, 25 with low-dose aspirin and calcium blockers, 16 with iloprost and/or bosentan, while the remaining 140 did not receive any drug.

Conclusion: Similar to adults, RP is more common in girls without an underlying disease. In patients with secondary RP, the symptoms. It has been shown that in patients with secondary RP, symptoms begin at a younger age and the ANA positivity and abnormal nailfold capillaries correlate.

REFERENCES:

[1] Jones G, et al. Arthritis Rheum 2003;48:3518-352

[2] Garner R et al. BMJ Open. 2015; 5(3): e006389

[3] Nigrovic PA et al. Pediatrics 2003, 111 (4) 715-721

Disclosure of Interests: None declared

DOI: 10.1136/annrheumdis-2021-eular.3076

\section{POS1320 DIFFERENCES IN CLINICAL MANIFESTATION AND DISEASE ACTIVITY OF PEDIATRIC BEHÇET DISEASE: A CROSS-SECTIONAL COHORT COMPARISON BETWEEN TURKEY AND UNITED STATES}

E. Demirkaya ${ }^{1}$, M. Romano ${ }^{1}$, C. Swearingen ${ }^{2}$, O. Kasapcopur ${ }^{3}$, B. Makay ${ }^{4}$, S. Özen ${ }^{5}$, Y. Yazici ${ }^{6} .{ }^{1}$ Schulich School of Medicine \& Dentistry, University of Western Ontario, Paediatric Rheumatology, LONDON, Canada; ${ }^{2}$ NYU Hospital for Joint Disease, Rheumatology, New YorK, United States of America; ${ }^{3}$ Istanbul University-Cerrahpasa, Paediatric Rheumatology, Instanbul, Turkey; ${ }^{4}$ Dokuz Eylul University, Paediatric Rheumatology, Izmir, Turkey; ${ }^{5}$ Hacettepe University, Ankara, Paediatric Rheumatology, Ankara, Turkey; ${ }^{6}$ New York University School of Medicine, Rheumatology, New York, United States of America

Background: Behçet disease (BD) is a systemic inflammatory disease which is rare in children. It is also known a higher prevalence along the ancient Silk Road and the eastern Mediterranean, especially in Turkey. Despite the presence of diagnostic criteria, the diagnosis of pediatric BD is still difficult due to atypical findings and the heterogeneity of the disease. While descriptive cohort studies in pediatric BD exist, direct comparison studies of clinical manifestations and disease activity between patients from different countries are scarce.

Objectives: We aimed to compare the main clinical features and activity of pediatric BD patients from Turkey versus United States (US).

Methods: The BD was diagnosed before 18 years of age and based on expert opinion. Disease activity was assessed with Physician Global Assessment (PhGA), Parents/Patient Global Assessment (PWPGA) and Behçet's Syndrome Activity Scale (BSAS) were administered to patients from both countries.

Results: A total of 161 patients were included (61 from Turkey; 100 from US). Males were more prevalent among patients from Turkey than among patients from US $(63.9 \%$ vs. $32 \%$, respectively, $p<0.001)$. Disease duration at the diagnosis was significantly longer in US $p=0.002$ ). Oral aphthosis was the most common symptom in both groups (96.7\% for Turkey and $73 \%$ for US), however a significant difference was found $(p<0.001)$. Genital ulcers were documented in $47 \%$ of US patients and in $41.4 \%$ of Turkish patients $(p=0.5)$. There were significant difference between two groups with regards to the ocular involvement: uveitis and posterior uveitis were more frequently in Turkish patients $(p=<0.001$ respectively) while retinal vasculitis was no seen in US patients $(p<0.001)$. Erythema nodosum occurred more frequently in Turkish group $(p<0.001)$. The pathergy test was reported as positive in 22 $(37.3 \%)$ patients in Turkey and $2(2.3 \%)$ in USA in our study $(p<0.001)$. Gastrointestinal and neurological involvement did not differ between cohorts. The rate of colchicine and oral steroids was similar. Azathioprine, cyclosporine and methotrexate usage was more frequent in Turkey $(p=0.005, p=0.007$, $p=0.033$, respectively) and infliximab was administered just in the US cohort $(p=0.002)$. PhGA and BSAS scores were higher in patients from Turkey $(p=0.003$ and $p=0.017$ respectively) and no significant differences were seen in PWPGA scores.

Conclusion: Disease activity and clinical features seem to be different between the two countries which may be linked to the environmental factors, referral patterns and immune system responses in the expression of this disease. The diagnosis of BD in the US was based on both fulfilling the ISBD criteria and in cases when criteria were not met, based on treating physician assessment which can explain some of the differences in disease presentation. In addition, there were differences in regard to clinical practice and treatment patterns between two countries. Increasing knowledge about heterogeneity of BD will improve the ability of diagnosis, development of new diagnostic criteria, and management of $\mathrm{BD}$.

\section{REFERENCES:}

[1] Butbul Aviel Y et al, Semin Arthritis Rheum. 2020

Disclosure of Interests: None declared

DOI: 10.1136/annrheumdis-2021-eular.3132 